\title{
EXPONENTIALLY ILL-CONDITIONED CONVECTION-DIFFUSION EQUATIONS IN MULTI-DIMENSIONAL DOMAINS
}

\author{
XIAODI SUN AND MICHAEL J. WARD*
}

\begin{abstract}
The phenomenon of dynamic metastability is analyzed for a class of singularly perturbed linear convection-diffusion equation in both a one-dimensional and a two-dimensional domain. The extreme sensitivity, known as a supersensitivity, of the time-dependent and steadystate solutions to exponentially small changes in the coefficient of the differential operator is also examined. Finally, some numerical experiments are performed in the one-dimensional case that indicate that it is possible to use standard finite difference methods to compute an exponentially ill-conditioned steady-state solution provided that quadruple precision arithmetic is used in the numerical computations.
\end{abstract}

1. Introduction. Certain linear time-dependent convection-diffusion equations with small diffusivity $\varepsilon$ exhibit the phenomenon of dynamic metastability whereby the time-dependent solution approaches a steady-state limit only over an asymptotically exponentially long time interval. In the limit $\varepsilon \rightarrow 0$, this metastable behavior and the corresponding steady-state limit can have an $O(1)$ sensitivity (i. e. a supersensitivity) to asymptotically exponentially small changes in the coefficient of the differential operator. This metastability, and the resulting supersensitivity, is analyzed for a certain class of linear singularly perturbed convection-diffusion equation in both a one and a two-dimensional spatial domain. In $\S 2$ we consider the one-dimensional case and in $\S 4$ we treat the corresponding two-dimensional problem. Some of the results for the one-dimensional problem have been given previously in [21].

For an exponentially ill-conditioned problem, an exponentially small change in the coefficient of the differential operator can have an $O(1)$ effect on the solution. Therefore, it is natural to expect that we might require that the truncation error associated with a numerical scheme for an exponentially ill-conditioned problem must be smaller than the magnitude of the exponentially small eigenvalue in order to ensure that the numerical solution resembles its continuous counterpart. With this heuristic reasoning, we might believe that high-order or spectral-type numerical methods are preferred for solving exponentially ill-conditioned problems. Such an approach, involving a high-order integral equation scheme, was implemented in [16] to compute boundary value resonance solutions, and a Galerkin spectral method was employed in [4] to compute metastable behavior for the viscous Cahn-Hilliard equation.

On the other hand, many conventional schemes have also been used to compute the solution to exponentially ill-conditioned problems. For instance, Elliott and French [9] studied metastable dynamics for the Cahn-Hilliard equation by applying the Galerkin finite element method, and Carr and Pego [7] computed the metastable evolution of a transition layer for the Allen-Cahn equation using the method of lines subroutine LSODI of the NAG library [20] with 801 grid points. In addition, a standard fourth-order finite difference scheme was employed to compute metastable dynamics for the viscous Cahn-Hilliard equation in [23]. However, the implementation in [23] was non-standard in that quadruple precision arithmetic was used in their numerical computations.

The main question that we consider is whether it is possible to accurately compute the solution to an exponentially ill-conditioned problem using a standard finite difference scheme employing quadruple rather than double precision arithmetic, or whether

*Department of Mathematics, University of British Columbia, Vancouver, B.C. V6T 1Z2, Canada. 
we must use a high order spectral method that ensures that the truncation error is smaller than the order of the exponentially small eigenvalue. In $\S 3$ we investigate this issue for a simple boundary layer resonance problem.

The study of metastability here complements previous studies of metastability for nonlinear reaction-diffusion problems undertaken in [2], [6], [7], [10], [14], [22], [23], [28], [29], and [30].

2. Ill-Conditioned Convection-Diffusion: One Dimension. In this section analyze an exponentially ill-conditioned linear convection-diffusion equation exhibiting metastable behavior in one dimension. The sensitivity of the steady-state solution to exponentially small changes in the coefficient of the differential operator is also examined. A related problem of this type was considered in [21].

We consider the following convection-diffusion equation for $u=u(x, t)$ :

$$
\begin{gathered}
u_{t}=\varepsilon u_{x x}-\Psi^{\prime}(x) u_{x}+\varepsilon^{\nu} g(x) e^{-a / \varepsilon} u, \quad-1<x<1, \quad t>0, \\
u(-1, t)=u_{l}, \quad u(1, t)=u_{r} ; \quad u(x, 0)=u_{0}(x) .
\end{gathered}
$$

Here $a>0, \nu, u_{l}$ and $u_{r}$ are constants, $\varepsilon \rightarrow 0^{+}$and $g(x), \Psi(x)$ and $u_{0}(x)$ are smooth. We assume that the potential $\Psi(x)$ has a global minimum on $[-1,1]$ at $x=0$ with $\Psi(0)=0, \Psi^{\prime}(0)=0$ and $\Psi^{\prime \prime}(0)>0$. Thus, the spatial operator in (2.1a) has a simple turning point at $x=0$. We also assume that $\Psi^{\prime}(x) \neq 0$ for $x \neq 0$ and thus $\Psi^{\prime}(1)>0$ and $\Psi^{\prime}(-1)<0$. Prototypical is $\Psi(x)=x^{2} / 2$.

When $g(x)=0$, the equilibrium problem corresponding to (2.1) and its associated eigenvalue problem arises in determining the exit time distribution for a Brownian particle confined by the potential well $\Psi$. Such problems have been well-studied in [17], [18], and [19] (see the references therein).

When $g(x)=0$, the eigenvalue problem associated with (2.1) is

$$
\begin{gathered}
L_{\varepsilon} \phi \equiv \varepsilon \phi_{x x}-\Psi^{\prime}(x) \phi_{x}=-\lambda \phi, \quad-1<x<1 ; \quad \phi( \pm 1)=0, \\
(\phi, \phi)_{w} \equiv \int_{-1}^{1} \phi^{2} w d x=1, \quad w \equiv e^{-\Psi / \varepsilon} .
\end{gathered}
$$

The eigenvalues $\lambda_{j}$ for $j \geq 0$ are real with $\lambda_{j}>0$ and the orthogonality relations $\left(\phi_{j}, \phi_{k}\right)_{w}=\delta_{j k}$ for $j, k=0,1, \ldots$, hold. It is well-known (cf. [8], [17], [16]) that $\lambda_{0}$ is exponentially small as $\varepsilon \rightarrow 0$ and has the asymptotic estimate

$$
\lambda_{0} \sim\left(\frac{\Psi^{\prime \prime}(0)}{2 \pi \varepsilon}\right)^{1 / 2}\left[\Psi^{\prime}(1) b_{r}(\varepsilon) e^{-\Psi(1) / \varepsilon}-\Psi^{\prime}(-1) b_{l}(\varepsilon) e^{-\Psi(-1) / \varepsilon}\right]
$$

Here $b_{r}(\varepsilon)$ and $b_{l}(\varepsilon)$ have asymptotic expansions in powers of $\varepsilon$ with leading terms $b_{r}(0)=b_{l}(0)=1$. To leading order, the corresponding eigenfunction $\phi_{0}$ has the boundary layer form

$$
\phi_{0} \sim M_{0}\left(1-e^{-\Psi^{\prime}(1)(1+x) / \varepsilon}-e^{\Psi^{\prime}(-1)(1-x) / \varepsilon}\right),
$$

where $M_{0}$ is a normalization constant. Notice that, as $\varepsilon \rightarrow 0, \phi_{0}$ is a constant away from the boundary layer regions.

We first consider the equilibrium problem for (2.1). For $\varepsilon \rightarrow 0$, a leading order boundary layer analysis for the equilibrium solution $U(x ; \varepsilon)$ shows that $U(x ; \varepsilon) \sim$ $\tilde{u}^{\varepsilon}\left[x ; A_{0 e}\right]$, where

$$
\tilde{u}^{\varepsilon}\left[x ; A_{0 e}\right] \equiv A_{0 e}+\left(u_{r}-A_{0 e}\right) e^{-\Psi^{\prime}(1)(1-x) / \varepsilon}+\left(u_{l}-A_{0 e}\right) e^{\Psi^{\prime}(-1)(1+x) / \varepsilon},
$$


for some undetermined constant $A_{0 e}$. Singular perturbation problems of this type, where a conventional application of the method of matched asymptotic expansions fails to select certain constants uniquely, were first identified in [1] and later studied extensively in [8], [11], [16], [26], and [32] (and the references therein). The relationship between this apparent indeterminacy and the ill-conditioning of the underlying operator is emphasized in [8] and [16]. More specifically, since (2.2a) has an exponentially small eigenvalue, and $L_{\varepsilon} \tilde{u}^{\varepsilon}$ is exponentially small away from the boundary layer regions near $x= \pm 1$ for any choice of $A_{0 e}$, it follows that the correct value of $A_{0 e}$ can only be determined by incorporating the effect of exponentially small terms into the asymptotic analysis. Methods for calculating $A_{0 e}$ are given in [11], [16], [19], and [26]. Since the equilibrium problem is exponentially ill-conditioned, it is natural to expect that it will be extremely sensitive to the exponentially small term in (2.1a). This aspect has been studied in [16], [26] and [32].

For the time-dependent problem for (2.1), we note that since $\lambda_{0}>0$, the equilibrium solution is stable when $a$ is sufficiently large. However, since $\lambda_{0}$ is exponentially small, the evolution of an arbitrary initial condition $u_{0}(x)$ to the equilibrium solution is exponentially slow. This metastable motion can be studied by using the projection method as developed in [21].

We now outline this method and some of the results obtained from it. Following [21], we seek a solution to (2.1) in the form

$$
u(x, t)=\tilde{u}^{\varepsilon}\left[x ; A_{0}(t)\right]+v(x, t),
$$

where $\tilde{u}^{\varepsilon}$ is defined in (2.5). Substituting (2.6) into (2.2), we obtain that $v(x, t)$ satisfies

$$
\begin{gathered}
v_{t}=L_{\varepsilon} v-\tilde{u}_{t}^{\varepsilon}+L_{\varepsilon} \tilde{u}^{\varepsilon}+\varepsilon^{\nu} g(x) e^{-a / \varepsilon}\left(\tilde{u}^{\varepsilon}+v\right), \quad-1<x<1, \quad t>0, \\
v(-1, t)=u_{l}-\tilde{u}^{\varepsilon}\left[-1 ; A_{0}(t)\right], \quad v(1, t)=u_{r}-\tilde{u}^{\varepsilon}\left[1 ; A_{0}(t)\right]
\end{gathered}
$$

together with the initial condition $v(x, 0)=u_{0}(x)-\tilde{u}^{\varepsilon}\left[x ; A_{0}(0)\right]$. We then expand $v(x, t)$ in terms of the eigenfunctions $\phi_{j}$ of $(2.2 \mathrm{a})-(2.2 \mathrm{~b})$ as

$$
v(x, t)=\sum_{j=0}^{\infty} c_{j}(t) \phi_{j}(x) .
$$

Using the orthogonality property of the eigenfunctions, we find that $c_{j}(t)$ satisfies the differential equation

$$
c_{j}^{\prime}+\lambda_{j} c_{j}=\left(\phi_{j}, L_{\varepsilon} \tilde{u}^{\varepsilon}\right)_{w}-\left.\varepsilon w v \phi_{j}^{\prime}\right|_{-1} ^{1}-\left(\phi_{j}, \tilde{u}_{t}^{\varepsilon}\right)_{w}+\varepsilon^{\nu} e^{-a / \varepsilon}\left(g \phi_{j}, \tilde{u}^{\varepsilon}+v\right)_{w}
$$

and has the initial value

$$
c_{j}(0)=\int_{-1}^{1}\left(u_{0}(x)-\tilde{u}^{\varepsilon}\left[x ; A_{0}(0)\right]\right) \phi_{j} w d x .
$$

Since $\lambda_{0}>0$ and is exponentially small, it is necessary that $c_{0}(t) \equiv 0$ in order to ensure that $v \ll \tilde{u}^{\varepsilon}$ over exponentially long time intervals . Therefore, the right-hand sides of (2.9) and (2.10) must vanish when $j=0$. Then, upon using $v \ll \tilde{u}^{\varepsilon}$ to simplify the last term on the right-hand side of (2.9), we obtain

$$
\left(\phi_{0}, \tilde{u}_{t}^{\varepsilon}\right)_{w} \sim\left(\phi_{0}, L_{\varepsilon} \tilde{u}^{\varepsilon}\right)_{w}-\left.\varepsilon w v \phi_{0}^{\prime}\right|_{-1} ^{1}+\varepsilon^{\nu} e^{-a / \varepsilon}\left(g \phi_{0}, \tilde{u}^{\varepsilon}\right)_{w}
$$


together with

$$
\int_{-1}^{1} \tilde{u}^{\varepsilon}\left[x ; A_{0}(0)\right] \phi_{0} w d x=\int_{-1}^{1} u_{0}(x) \phi_{0} w d x .
$$

Equation (2.11) is a differential equation for $A_{0}(t)$ and (2.12) determines the initial value $A_{0}(0)$.

To obtain an explicit differential equation for $A_{0}$ we use the form for $\phi_{0}$ given in (2.4) to evaluate the various terms in (2.11)-(2.12) asymptotically for $\varepsilon \rightarrow 0$ (see [21] for details). To leading order as $\varepsilon \rightarrow 0$, the analysis in [21] shows that $A_{0}(t)$ satisfies the limiting differential equation

$$
A_{0}^{\prime} \sim-\left(\lambda_{0}-\varepsilon^{\nu} g(0) e^{-a / \varepsilon}\right) A_{0}+\beta\left[u_{r} \Psi^{\prime}(1) e^{-\Psi(1) / \varepsilon}-u_{l} \Psi^{\prime}(-1) e^{-\Psi(-1) / \varepsilon}\right]
$$

with

$$
A_{0}(0) \sim u_{0}(0), \quad \text { where } \quad \beta \equiv\left[\Psi^{\prime \prime}(0) /(2 \pi \varepsilon)\right]^{1 / 2} .
$$

In (2.13a) $\lambda_{0}$ is given in (2.3). The metastable dynamics for $u(x, t)$ is then given by $u(x, t) \sim \tilde{u}^{\varepsilon}\left[x ; A_{0}(t)\right]$, where $\tilde{u}^{\varepsilon}$ is defined in (2.5). If $a$ is sufficiently large, it follows that $A_{0}(t) \rightarrow A_{0 e}$ as $t \rightarrow \infty$, where $A_{0 e}$ is obtained by setting $A_{0}^{\prime}=0$ in (2.13a). Higher order corrections to the asymptotic differential equation for $A_{0}(t)$ are given in [21].

2.1. An Example. We let $\Psi(x)=x^{2} / 2, g(x)=g_{0} e^{-x^{2} / 2}$, and $\nu=-1 / 2$. Then, from (2.3) we calculate that

$$
\lambda_{0} \sim\left(\frac{2}{\pi \varepsilon}\right)^{1 / 2} e^{-\varepsilon^{-1} / 2}
$$

From (2.5), (2.6), and (2.13a), we obtain that for $t \gg 1$,

$$
u(x, t) \sim A_{0}(t)+\left[u_{r}-A_{0}(t)\right] e^{-(1-x) / \varepsilon}+\left[u_{l}-A_{0}(t)\right] e^{-(1+x) / \varepsilon},
$$

where $A_{0}(t)$ satisfies the asymptotic differential equation

$$
A_{0}^{\prime} \sim-\left(\frac{2}{\pi \varepsilon}\right)^{1 / 2} e^{-\varepsilon^{-1} / 2}\left[\left(1-\left(\frac{\pi}{2}\right)^{1 / 2} g_{0} e^{-(a-1 / 2) / \varepsilon}\right) A_{0}-\frac{\left(u_{l}+u_{r}\right)}{2}\right]
$$

Now, from (2.16) we observe that when

$$
\left(\frac{\pi}{2}\right)^{1 / 2} g_{0} e^{-(a-1 / 2) / \varepsilon}<1
$$

then $A_{0}(t) \rightarrow A_{0 e}$ as $t \rightarrow \infty$, where

$$
A_{0 e}=\frac{\left(u_{l}+u_{r}\right)}{2}\left[1-\left(\frac{\pi}{2}\right)^{1 / 2} g_{0} e^{-(a-1 / 2) / \varepsilon}\right]^{-1} .
$$

Thus, if $a>1 / 2$, so that the order of the exponentially small perturbing term $\varepsilon^{\nu} g(x) e^{-a / \varepsilon}$ is smaller than that of the exponentially small eigenvalue, then $A_{0}(t) \rightarrow$ 
$A_{0 e}$ as $t \rightarrow \infty$ for any $A_{0}(0)$. However, if $0<a<1 / 2$, then $A_{0}(t) \rightarrow A_{0 e}$ as $t \rightarrow \infty$ only when $g_{0} \leq 0$. In the borderline case where $a=1 / 2$, then

$$
\lim _{t \rightarrow \infty} A_{0}(t)=A_{0 e}, \quad \text { when } \quad g_{0}<(2 / \pi)^{1 / 2},
$$

and $A_{0}(t) \rightarrow \infty$ as $t \rightarrow \infty$ when $g_{0}>(2 / \pi)^{1 / 2}$. This example clearly shows the effect of supersensitivity, whereby the exponentially small perturbing term can have an $O(1)$ effect on both the time-dependent and the steady-state solutions.

3. Some Numerical Experiments in One Dimension. In this section we numerically study the convergence properties of a few numerical schemes to compute solutions to an exponentially ill-conditioned boundary value problem. Our goal is to give some guidelines in designing numerical schemes to treat ill-conditioned problems. The main question we would like to address is whether it is possible to accurately compute the solution to an exponentially ill-conditioned problem using a standard finite difference method employing quadruple, or extended, precision arithmetic, instead of using a higher order spectral method.

For simplicity, we investigate this numerical issue for the following singularly perturbed boundary value resonance problem

$$
\begin{gathered}
L_{\varepsilon} u \equiv \varepsilon u_{x x}-x^{2 m+1} p(x) u_{x}=0, \quad-1<x<1, \\
u(-1)=u_{l}, \quad u(1)=u_{r} .
\end{gathered}
$$

Here $\varepsilon>0$ is a small parameter, $m \geq 0$ is an integer and $p(x)>0$ on $[-1,1]$. One main reason to consider (3.1) is that it is linear and its solution can be found analytically. Thus, we can easily compute the errors associated with the finite difference schemes.

We now introduce the three finite difference schemes used to compute solutions to (3.1). The mesh is denoted by $I^{h}=\left\{x_{i}:-1=x_{N}<x_{-N+1}<\ldots<x_{N-1}<x_{N}=1\right\}$ with mesh widths $h_{i}=x_{i}-x_{i-1}$ and $h=\max _{i} h_{i}$. Two meshes will be used in the computations.

- An equidistant mesh $I_{e}^{h}=\left\{x_{i}=i h: i=-N, \ldots, N, N h=1\right\}$.

- A non-equidistant mesh $I_{n}^{h}=\left\{x_{i}: i=-N, \ldots, N\right\}$, where with $h=1 / N$,

$$
x_{i}= \begin{cases}\lambda(i h+1)-1, & i=-N, \ldots, 0 \\ 1-\lambda(1-i h), & i=1, \ldots, N\end{cases}
$$

Here the mesh generating function $\lambda(t)$ is defined by

$$
\lambda(t)= \begin{cases}\psi(t):=-a \varepsilon \ln (1-t / q), & t \in[0, \alpha], \\ \pi(t):=\psi(\alpha)+\psi^{\prime}(\alpha)(t-\alpha), & t \in(\alpha, 1],\end{cases}
$$

where $q \in(0,1)$ and $a>0$ are constants, and $\alpha \in(0, q)$ is determined uniquely by $\pi(1)=1$. In the computations below we chose $a=2, q=0.5$, for which $\alpha=0.4618$. This mesh is also called the Bakhvalov mesh [3], and it allows for more mesh points near the endpoints to resolve boundary layers. Using the usual notation for divided differences,

$$
\begin{array}{ll}
D_{+} u_{i}=\left(u_{i+1}-u_{i}\right) / h_{i+1}, & D_{-} u_{i}=\left(u_{i}-u_{i-1}\right) / h_{i}, \\
D_{0} u_{i}=\left(u_{i+1}-u_{i-1}\right) /\left(h_{i}+h_{i+1}\right), & D_{+} D_{-} u_{i}=2\left(D_{+} u_{i}-D_{-} u_{i}\right) /\left(h_{i}+h_{i+1}\right)
\end{array}
$$

we define the following three finite difference schemes: 
- The upwind scheme on the non-equidistant mesh $I_{n}^{h}$,

$$
L_{1}^{h} u_{i}=\varepsilon D_{+} D_{-} u_{i}+P_{i} D^{\prime} u_{i}=0,-N<i<N, u_{-N}=u_{l}, u_{N}=u_{r},
$$

where $P_{i}=-x_{i}^{2 m+1} p\left(x_{i}\right)$, and

$$
D^{\prime} u_{i}= \begin{cases}D_{+} u_{i}, & \text { if } P_{i} \geq 0 \\ D_{-} u_{i}, & \text { if } P_{i}<0\end{cases}
$$

- The coupled scheme on the non-equidistant mesh $I_{n}^{h}$,

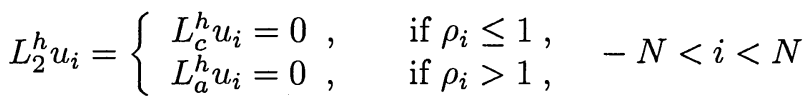

$$
\begin{aligned}
& u_{-N}=u_{l}, \quad u_{N}=u_{r}
\end{aligned}
$$

where

$$
\begin{aligned}
& \rho_{i}= \begin{cases}h_{i} P_{i} / 2 \varepsilon, & \text { if } P_{i} \geq 0, \\
-h_{i+1} P_{i} / 2 \varepsilon, & \text { if } P_{i}<0,\end{cases} \\
& L_{c}^{h} u_{i}=\varepsilon D_{+} D_{-} u_{i}+P_{i} D_{0} u_{i}, \quad \text { (central scheme [24]), }
\end{aligned}
$$

$$
L_{a}^{h} u_{i}=\varepsilon D_{+} D_{-} u_{i}+P_{i \pm \frac{1}{2}} D_{ \pm} u_{i}, \quad \text { (Gushchin-Shchennikow scheme [12]) . }
$$

Here we use the plus sign (minus sign) in $D_{ \pm}$if $P_{i}$ is positive (negative).

- The Il'in scheme on the equidistant mesh $I_{e}^{h}$,

$$
L_{3}^{h} u_{i}=\varepsilon \sigma_{i} D_{+} D_{-} u_{i}+P_{i} D_{0} u_{i}=0, \quad-N<i<N, u_{-N}=u_{l}, u_{N}=u_{r},
$$

where the fitting factor $\sigma_{i}$ is $\sigma_{i} \equiv \frac{P_{i} h}{2 \varepsilon} \operatorname{coth} \frac{P_{i} h}{2 \varepsilon}$.

The background and motivation behind these finite difference schemes and meshes can be found in [3], [13], [15], [24], [25], [31], and the references therein.

Numerical results for the coupled scheme computed using double precision arithmetic when $p(x)=1, m=0, u_{l}=-3$ and $u_{r}=1$ are shown in Table 3.1. Since the exact solution $u(x)$ to (3.1) is known we can easily compute the error for different values of $h$ and $\varepsilon$. The results in this table indicate that the coupled scheme is uniformly second order convergent in the parameter $\varepsilon$ only when the number $N$ of mesh points is not too large. On the contrary, for large $N$ the convergence property of the scheme deteriorates, contradicting classical convergence theory that states that the numerical error of a convergent scheme will tend to zero as the mesh size $h \rightarrow 0$. Our explanation of this paradox is that the three schemes do converge uniformly in $\varepsilon$, but that the degeneration for large $N$ is caused by the round-off errors of a computer due to the exponential ill-conditioning of the finite difference operators. To verify this explanation, we show the numerically computed minimum and maximum eigenvalues and the condition numbers of the coefficient matrixes of the coupled scheme in Table 3.2 for $\varepsilon=0.02$ and for different mesh size $h$. Comparing the results in Table 3.2 with Table 3.1, we notice that the numerical convergence order $\gamma$ begins to deviate from the analytical value of 2 only when the condition number reaches $O\left(10^{15}\right)$, which is approximately the reciprocal of the (double) machine precision. Thus, we believe that 


\begin{tabular}{|l|c|c|c|c|c|c|}
\hline \hline \multirow{2}{*}{$N$} & \multicolumn{2}{|c|}{$\varepsilon=0.03$} & \multicolumn{2}{c|}{$\varepsilon=0.02$} & \multicolumn{2}{c|}{$\varepsilon=0.01$} \\
\cline { 2 - 7 } & $E_{\infty}^{h}$ & $\gamma$ & $E_{\infty}^{h}$ & $\gamma$ & $E_{\infty}^{h}$ & $\gamma$ \\
\hline 12 & $.2545 \mathrm{e}-1$ & 2.41 & $.2184 \mathrm{e}-1$ & 1.89 & $.1900 \mathrm{e}-1$ & 2.04 \\
24 & $.4789 \mathrm{e}-2$ & 2.00 & $.5872 \mathrm{e}-2$ & 2.15 & $.4615 \mathrm{e}-2$ & -7.77 \\
48 & $.1199 \mathrm{e}-2$ & 1.99 & $.1319 \mathrm{e}-2$ & 1.87 & 1.01 & - \\
96 & $.3017 \mathrm{e}-3$ & 1.98 & $.3598 \mathrm{e}-3$ & 0.22 & - & - \\
192 & $.7659 \mathrm{e}-4$ & 1.99 & $.3088 \mathrm{e}-3$ & -0.25 & - & - \\
384 & $.1925 \mathrm{e}-4$ & 1.79 & $.1466 \mathrm{e}-2$ & -1.10 & - & - \\
768 & $.5558 \mathrm{e}-5$ & -0.76 & $.3136 \mathrm{e}-2$ & -0.97 & - & - \\
1536 & $.9406 \mathrm{e}-5$ & - & $.6162 \mathrm{e}-2$ & - & - & - \\
\hline
\end{tabular}

Numerical results of the coupled scheme for (3.1) with $p(x)=1, m=0, u_{l}=-3$, and $u_{r}=1$, using double precision arithmetic. Here, $E_{\infty}^{h} \equiv \max _{i}\left|u\left(x_{i}\right)-u_{i}\right|$ is the maximum error of the numerical solution on a mesh with $h=1 / N, \gamma$ is the numerical convergence order defined by $\gamma=\left(\log E_{\infty}^{h}-\log E_{\infty}^{h / 2}\right) / \log 2$. Note that from (2.3), the principal eigenvalue of the operator in (3.1) satisfies $\lambda_{0} \sim 2.662 \times 10^{-7}$ for $\varepsilon=0.03, \lambda_{0} \sim 7.835 \times 10^{-11}$ for $\varepsilon=0.02$, and $\lambda_{0} \sim 1.539 \times 10^{-21}$ for $\varepsilon=0.01$.

\begin{tabular}{|l|c|c|c|}
\hline \hline $\mathrm{N}$ & $\lambda_{\min }$ & $\lambda_{\max }$ & Cond \\
\hline 12 & $3.633 \mathrm{e}-7$ & 778.0 & $4.866 \mathrm{e} 9$ \\
24 & $3.116 \mathrm{e}-10$ & $4.414 \mathrm{e} 3$ & $3.199 \mathrm{e} 13$ \\
48 & $8.186 \mathrm{e}-11$ & $2.141 \mathrm{e} 4$ & $6.314 \mathrm{e} 14$ \\
96 & $4.450 \mathrm{e}-11$ & $9.595 \mathrm{e} 4$ & $4.483 \mathrm{e} 15$ \\
192 & $7.193 \mathrm{e}-11$ & $4.113 \mathrm{e} 5$ & $1.159 \mathrm{e} 16$ \\
384 & $1.068 \mathrm{e}-11$ & $1.717 \mathrm{e} 6$ & $5.003 \mathrm{e} 16$ \\
\hline \multicolumn{4}{|c|}{ TABLE 3.2}
\end{tabular}

Numerical results (using Matlab) of the minimum eigenvalue $\lambda_{\min }$, the maximum eigenvalue $\lambda_{\max }$, and the condition number Cond of the coefficient matrix of the coupled scheme (3.5) when $\varepsilon=0.02$. The other parameters are the same as in Table 3.1.

this degeneration of the convergence can be avoided if we can calculate the discrete scheme more accurately. Based on this explanation, it is anticipated that numerical computations with quadruple precision arithmetic will yield better results than those with double precision. This is verified in Table 3.3, where first order convergence for the upwind scheme and second order convergence for the coupled scheme and Il'in scheme are obtained using quadruple precision arithmetic even for much larger $N$ than those in Table 3.1.

In summary, these numerical experiments indicate that for an exponentially illconditioned problem, a truncation error $\tau^{h}$ may not lead to very large errors in the numerical solution $u^{h}$ and a scheme may still be uniformly convergent with respect to $\varepsilon$. A rigorous analytical proof of the uniform convergence of the three schemes described above for (3.1) is given in [27]. However, a numerical method will usually inherit the ill-conditioning associated with the underlying continuous problem. This causes the peculiar phenomenon observed in the computations above that a small grid size $N$ may give better numerical results than a larger value of $N$. To minimize the effects of round-off errors which may pollute the accuracy of a scheme, higher (such as quadruple) precision arithmetic is preferred in solving the discrete schemes. This heuristic explanation perhaps suggests why the quadruple precision computations of metastability in the viscous Cahn-Hilliard done in [23], while employing a standard 


\begin{tabular}{|l|c|c|c|c|c|c|}
\hline \hline \multirow{2}{*}{$N$} & \multicolumn{2}{|c|}{ Upwind scheme (3.4) } & \multicolumn{2}{c|}{ Coupled scheme (3.5) } & \multicolumn{2}{c|}{ Il'in scheme (3.6) } \\
\cline { 2 - 7 } & $E_{\infty}^{h}$ & $\gamma$ & $E_{\infty}^{h}$ & $\gamma$ & $E_{\infty}^{h}$ & $\gamma$ \\
\hline 48 & $.8204 \mathrm{e}-1$ & 0.98 & $.1225 \mathrm{e}-2$ & 1.89 & $.1815 \mathrm{e}-2$ & 1.45 \\
96 & $.4153 \mathrm{e}-1$ & 0.99 & $.3310 \mathrm{e}-3$ & 2.00 & $.6603 \mathrm{e}-3$ & 1.97 \\
192 & $.2084 \mathrm{e}-1$ & 1.00 & $.8265 \mathrm{e}-4$ & 2.00 & $.1675 \mathrm{e}-3$ & 1.99 \\
384 & $.1044 \mathrm{e}-1$ & 1.00 & $.2064 \mathrm{e}-4$ & 2.00 & $.4221 \mathrm{e}-4$ & 1.97 \\
768 & $.5222 \mathrm{e}-2$ & 1.00 & $.5154 \mathrm{e}-5$ & 2.00 & $.1075 \mathrm{e}-4$ & 1.90 \\
1536 & $.2611 \mathrm{e}-2$ & - & $.1286 \mathrm{e}-5$ & - & $.2875 \mathrm{e}-5$ & - \\
\hline
\end{tabular}

Numerical results of the three schemes using quadruple precision arithmetic. Here, $\varepsilon=0.01$, and the other parameters are the same as in Table 3.1.

finite difference scheme, was successful.

4. Ill-Conditioned Convection-Diffusion: Two Dimensions. We now generalize (2.1) to the case of two spatial dimensions. To this end we consider the following convection-diffusion equation in a bounded two-dimensional domain $D$ with a smooth boundary $\partial D$ :

$$
\begin{gathered}
u_{t}=\varepsilon \Delta u-\nabla \Psi \cdot \nabla u+\varepsilon^{\nu} g(\mathbf{x}) e^{-a / \varepsilon} u, \quad \mathbf{x} \in D, \quad t>0 \\
u=u_{b}(\mathbf{x}), \quad \mathbf{x} \in \partial D ; \quad u(\mathbf{x}, 0)=u_{0}(\mathbf{x}) .
\end{gathered}
$$

Here $u=u(\mathbf{x}, t)$ with $\mathbf{x}=\left(x_{1}, x_{2}\right), \nu$ and $a>0$ are constants, $\varepsilon \rightarrow 0^{+}$, and $g(\mathbf{x})$, $u_{b}(\mathbf{x})$ and $u_{0}(\mathbf{x})$ are smooth. Let $\bar{D}=D \cup \partial D$. We assume that the potential $\Psi(\mathbf{x})$ has a unique global minimum on $\bar{D}$ at some interior point $\mathrm{x}_{0} \in D$. At this minimum point we assume that

$$
\Psi\left(\mathbf{x}_{0}\right)=0, \quad \nabla \Psi\left(\mathbf{x}_{0}\right)=0, \quad H\left[\Psi\left(\mathbf{x}_{0}\right)\right]>0,
$$

where $H(\Psi) \equiv \Psi_{x_{1} x_{1}} \Psi_{x_{2} x_{2}}-\Psi_{x_{1} x_{2}}^{2}$ is the Hessian. We also assume that $\nabla \Psi(\mathbf{x}) \neq 0$ for $\mathbf{x} \neq \mathbf{x}_{0}$ and that $\nabla \Psi \cdot \hat{n}>0$ on $\partial D$, where $\hat{n}$ is the unit outward normal to $\partial D$.

In place of (2.2), the eigenvalue problem associated with (4.1) when $g(\mathbf{x}) \equiv 0$ is

$$
\begin{gathered}
L_{\varepsilon} \phi \equiv \varepsilon \Delta \phi-\nabla \Psi \cdot \nabla \phi=-\lambda \phi, \quad \mathbf{x} \in D ; \quad \phi=0, \quad \mathbf{x} \in \partial D, \\
(\phi, \phi)_{w} \equiv \int_{D} \phi^{2} w d \mathbf{x}=1, \quad w \equiv e^{-\Psi / \varepsilon} .
\end{gathered}
$$

The eigenvalues $\lambda_{j}$ for $j \geq 0$ are real with $\lambda_{j}>0$ and $\left(\phi_{j}, \phi_{k}\right)_{w}=\delta_{j k}$ for $j, k=0,1, \ldots$

4.1. Calculation of the Principal Eigenvalue. We now asymptotically calculate the principal eigenvalue $\lambda_{0}$ as $\varepsilon \rightarrow 0$ for three different cases. The corresponding eigenfunction has the boundary layer form

$$
\phi_{0} \sim M_{0}\left(1-e^{\gamma \eta / \varepsilon}\right), \quad \text { where } \quad \gamma=\left.\gamma(s) \equiv \nabla \Psi \cdot \hat{n}\right|_{\partial D}>0
$$

and $M_{0}$ is a normalization constant. Here $s$ denotes arclength along $\partial D,-\eta$ is the distance from $\mathbf{x} \in D$ to $\partial D$, and $\hat{n}$ is the unit outward normal to $\partial D$. Since $\gamma>0$, then $\phi_{0} \rightarrow M_{0}$ as $\eta / \varepsilon \rightarrow-\infty$. To calculate $\lambda_{0}$, we integrate by parts to obtain the following key identity valid for any $u$ and $v$ :

$$
\left(L_{\varepsilon} u, v\right)_{w}-\left(u, L_{\varepsilon} v\right)_{w}=\int_{\partial D} \varepsilon v e^{-\Psi / \varepsilon} \partial_{n} u d s-\int_{\partial D} \varepsilon u e^{-\Psi / \varepsilon} \partial_{n} v d s
$$


Here $\partial_{n}$ denotes the outward normal derivative. Substituting $u=\phi_{0}$ and $v=1$ into (4.5), we obtain

$$
\lambda_{0}\left(\phi_{0}, 1\right)=-\int_{\partial D} \varepsilon e^{-\Psi / \varepsilon} \partial_{n} \phi_{0} d s .
$$

Next, since the dominant contribution to the left-hand side of (4.6) arises from the region near $\mathbf{x}=\mathbf{x}_{0}$ where $\Psi$ has a non-degenerate minimum, we obtain for $\varepsilon \rightarrow 0$ that

$$
\left(\phi_{0}, 1\right)_{w} \sim 2 \pi \varepsilon M_{0}\left(H\left[\Psi\left(\mathbf{x}_{0}\right)\right]\right)^{-1 / 2} .
$$

Here $H$ is the Hessian of $\Psi$ at $\mathbf{x}_{0}$ defined following (4.2) above. Substituting (4.4) and (4.7) into (4.6), we obtain the following estimate for $\lambda_{0}$ as $\varepsilon \rightarrow 0$ :

$$
\lambda_{0} \sim \frac{\left(H\left[\Psi\left(\mathbf{x}_{0}\right)\right]\right)^{1 / 2}}{2 \pi \varepsilon} \int_{\partial D} e^{-\Psi / \varepsilon} \nabla \Psi \cdot \hat{n} d s .
$$

Equation (4.8) shows that $\lambda_{0}$ is determined by the precise behavior of $\Psi$ on $\partial D$.

Let $\left(x_{1}, x_{2}\right)=\left(x_{1}(s), x_{2}(s)\right)$ be a parametric representation of $\partial D$ where $s$ denotes arclength on $\partial D$ and $\tilde{\Psi}(s)=\Psi\left(x_{1}(s), x_{2}(s)\right)$. In calculating $\lambda_{0}$ asymptotically, we distinguish three cases:

Case 1: Suppose that the minimum value of $\tilde{\Psi}$ on $\partial D$ is taken at $N$ distinct points $\mathbf{y}_{j} \in \partial D$, with arclength coordinate $s_{j}$, for $j=1, . ., N$. Assume that these minima are non-degenerate. Then, the principal eigenvalue $\lambda_{0}$ of (4.3) is exponentially small as $\varepsilon \rightarrow 0$ and has the asymptotic estimate

$$
\lambda_{0} \sim\left(\frac{H\left[\Psi\left(\mathbf{x}_{0}\right)\right]}{2 \pi \varepsilon}\right)^{1 / 2} e^{-\Psi^{*} / \varepsilon} \sum_{j=1}^{N}\left|\nabla \Psi\left(\mathbf{y}_{j}\right)\right|\left(\tilde{\Psi}^{\prime \prime}\left(s_{j}\right)\right)^{-1 / 2} .
$$

Here $\Psi^{*} \equiv \Psi\left(\mathbf{y}_{j}\right)$ for $j=1, . ., N$, and

$$
\left.\tilde{\Psi}^{\prime \prime}\left(s_{j}\right) \equiv|\nabla \Psi|^{-2}\left[\Psi_{x_{1} x_{1}} \Psi_{x_{2}}^{2}-2 \Psi_{x_{1} x_{2}} \Psi_{x_{1}} \Psi_{x_{2}}+\Psi_{x_{2} x_{2}} \Psi_{x_{1}}^{2}+\kappa_{j}|\nabla \Psi|^{3}\right]\right|_{\mathbf{x}=\mathbf{y}_{j}},
$$

where $\kappa_{j}<0$ is the curvature of $\partial D$ at $\mathbf{y}_{j}$.

Case 2: Suppose that the minimum value $\Psi^{\star}$ of $\tilde{\Psi}$ on $\partial D$ is achieved at the points $s_{1}, s_{2}, \ldots, s_{N}$ and that $\tilde{\Psi}(s)-\tilde{\Psi}\left(s_{j}\right)=d_{j}^{-2 p}\left(s-s_{j}\right)^{2 p}(1+o(1))$ as $s \rightarrow s_{j}$ where $d_{j}>0(j=1,2, \ldots, N)$ and $p$ is a positive integer. Then, in this case where we allow for the minima to be degenerate, we get for $\varepsilon \rightarrow 0$ that

$$
\lambda_{0} \sim \frac{\Gamma\left(\frac{1}{2 p}\right)}{2 \pi p} \varepsilon^{\frac{1}{2 p}-1}\left(H\left[\Psi\left(\mathbf{x}_{0}\right)\right]\right)^{\frac{1}{2}} e^{-\Psi^{\star} / \varepsilon} \sum_{j=1}^{N} d_{j}\left|\nabla \Psi\left(\mathbf{x}_{j}\right)\right| .
$$

Here $\mathbf{x}_{j}=\left(x_{1}\left(s_{j}\right), x_{2}\left(s_{j}\right)\right)$ for $j=1,2, \ldots, N$.

Case 3: Suppose that the maximum value $\Psi^{\star}$ of $\Psi$ on $\partial D$ is achieved on a set $S \subset \partial D$, with a nonempty interior $S^{0}$ and that the measure of $S-S^{0}$ in $\partial D$ is zero. Then, for $\varepsilon \rightarrow 0$, we have the asymptotic estimate

$$
\lambda_{0} \sim \frac{1}{2 \pi \varepsilon}\left(H\left[\Psi\left(\mathbf{x}_{0}\right)\right]\right)^{\frac{1}{2}} e^{-\Psi^{*} / \varepsilon} \int_{S}|\nabla \Psi| d s .
$$


4.2. Derivation of the Metastability Result. For $\varepsilon \rightarrow 0$, a leading order boundary layer approximation for the equilibrium solution $U(\mathbf{x} ; \varepsilon)$ to $(4.1)$ is given by

$$
U(\mathbf{x} ; \varepsilon) \sim \tilde{u}^{\varepsilon}\left[\mathbf{x} ; A_{0 e}\right] \equiv A_{0 e}+\left[u_{b}(s)-A_{0 e}\right] e^{\gamma \eta / \varepsilon},
$$

in an $O(\varepsilon)$ neighborhood near $\partial D$. Here we have written the boundary data $u_{b}$ in terms of an arclength coordinate $s$. The constant $A_{0 e}$ in (4.12) is undetermined. This indeterminacy is caused by the exponential ill-conditioning of the elliptic operator in (4.1).

For the special case $g(\mathbf{x})=0$, methods to calculate $A_{0 e}$ were given in [11] and [18]. In this case, since the principal eigenvalue $\lambda_{0}$ is positive, the equilibrium solution to (4.1) is stable. Starting from any initial value $u_{0}(\mathbf{x})$, a quasi-equilibrium solution to (4.1) of the spatial form (4.12) will be obtained on an $O(1)$ time scale. Once this quasi-equilibrium solution has been formed, it will approach its stable equilibrium solution over an exponentially long time scale.

To study this metastable motion analytically for an arbitrary $g(\mathbf{x})$ we set $u(\mathbf{x}, t)=$ $\tilde{u}^{\varepsilon}\left[\mathbf{x}, A_{0}(t)\right]+v(\mathbf{x}, t)$ in (4.1) and obtain that $v(\mathbf{x}, t)$ satisfies

$$
\begin{aligned}
& v_{t}=L_{\varepsilon} v-\tilde{u}_{t}^{\varepsilon}+L_{\varepsilon} \tilde{u}^{\varepsilon}+\varepsilon^{\nu} g(\mathbf{x}) e^{-a / \varepsilon}\left(\tilde{u}^{\varepsilon}+v\right), \quad \mathbf{x} \in D, \quad t>0 \\
& v=u_{b}-\tilde{u}^{\varepsilon}, \quad \mathbf{x} \in \partial D ; v(\mathbf{x}, 0)=u_{0}(\mathbf{x})-\tilde{u}^{\varepsilon}\left[\mathbf{x} ; A_{0}(0)\right], \quad \mathbf{x} \in D
\end{aligned}
$$

We then expand $v$ in terms of the eigenfunctions $\phi_{j}$ of (4.3) as

$$
v(\mathbf{x}, t)=\sum_{j=0}^{\infty} c_{j}(t) \phi_{j}(\mathbf{x})
$$

Using the othogonality properties of the eigenfunctions, we obtain that $c_{j}(t)$ satisfies

$$
c_{j}^{\prime}+\lambda_{j} c_{j}=\left(\phi_{j}, L_{\varepsilon} \tilde{u}^{\varepsilon}\right)_{w}-\int_{\partial D} \varepsilon w v \partial_{n} \phi_{j} d s-\left(\phi_{j}, \tilde{u}_{t}^{\varepsilon}\right)_{w}+\varepsilon^{\nu} e^{-a / \varepsilon}\left(g \phi_{j}, \tilde{u}^{\varepsilon}+v\right)_{w},
$$

with the initial value

$$
c_{j}(0)=\int_{D}\left(u_{0}(\mathbf{x})-\tilde{u}^{\varepsilon}\left[\mathbf{x} ; A_{0}(0)\right]\right) \phi_{j} w d \mathbf{x} .
$$

Here $\partial_{n}$ denotes the outward normal derivative to $\partial D$. Since $\lambda_{0}>0$ and is exponentially small, it is necessary that $c_{0}(t) \equiv 0$ in order to ensure that $v \ll \tilde{u}^{\varepsilon}$ over exponentially long time intervals. Thus we require that the right-hand sides of (4.15a) and (4.15b) vanish when $j=0$. This leads to the following differential equation for $A_{0}(t)$

$$
\left(\phi_{0}, \tilde{u}_{t}^{\varepsilon}\right)_{w} \sim\left(\phi_{0}, L_{\varepsilon} \tilde{u}^{\varepsilon}\right)_{w}-\int_{\partial D} \varepsilon w v \partial_{n} \phi_{0} d s+\varepsilon^{\nu} e^{-a / \varepsilon}\left(g \phi_{0}, \tilde{u}^{\varepsilon}\right)_{w}
$$

together with the initial condition

$$
\int_{D} \tilde{u}^{\varepsilon}\left[x ; A_{0}(0)\right] \phi_{0} w d x=\int_{D} u_{0}(x) \phi_{0} w d x .
$$


To obtain an explicit ordinary differential equation for $A_{0}(t)$, we use the form (4.12) to asymptotically evaluate the various terms in (4.16) for $\varepsilon \rightarrow 0$. We first use the identity (4.5) to calculate

$$
\left(\phi_{0}, L_{\varepsilon} \tilde{u}^{\varepsilon}\right)_{w}=-\lambda_{0}\left(\tilde{u}^{\varepsilon}, \phi_{0}\right)_{w}-\int_{\partial D} \varepsilon \tilde{u}^{\varepsilon} w \partial_{n} \phi_{0} d s .
$$

Substituting (4.17) into (4.16a), we obtain

$$
\left(\phi_{0}, \tilde{u}_{t}^{\varepsilon}\right)_{w} \sim-\lambda_{0}\left(\tilde{u}^{\varepsilon}, \phi_{0}\right)_{w}+\varepsilon^{\nu} e^{-a / \varepsilon}\left(\tilde{u}^{\varepsilon}, g \phi_{0}\right)_{w}-\int_{\partial D} \varepsilon e^{-\Psi / \varepsilon} u_{b} \partial_{n} \phi_{0} d s .
$$

Next, the inner products over $D$ are readily evaluated using Laplace's method since the dominant contribution to each of them arises from the region near $\mathbf{x}=\mathbf{x}_{0}$. For $\varepsilon \rightarrow 0$, we calculate that

$$
\begin{aligned}
&\left(\phi_{0}, \tilde{u}_{t}^{\varepsilon}\right)_{w} \sim 2 \pi \varepsilon A_{0}^{\prime}\left(H\left[\Psi\left(\mathbf{x}_{0}\right)\right]\right)^{-1 / 2} \\
&\left(\tilde{u}^{\varepsilon}, \phi_{0}\right)_{w} \sim 2 \pi \varepsilon A_{0}\left(H\left[\Psi\left(\mathbf{x}_{0}\right)\right]\right)^{-1 / 2} \\
&\left(\tilde{u}^{\varepsilon}, g \phi_{0}\right)_{w} \sim 2 \pi \varepsilon A_{0} g\left(\mathbf{x}_{0}\right)\left(H\left[\Psi\left(\mathbf{x}_{0}\right)\right]\right)^{-1 / 2}
\end{aligned}
$$

Here $A_{0}^{\prime} \equiv d A_{0} / d t$. Substituting (4.19) into (4.18), and calculating the boundary integral in (4.18) asymptotically (as in the calculation of $\lambda_{0}$ ), we obtain that $A_{0}(t)$ satisfies the following limiting differential equation for $\varepsilon \rightarrow 0$ :

$$
\begin{gathered}
A_{0}^{\prime} \sim-\left(\lambda_{0}-\varepsilon^{\nu} g\left(\mathbf{x}_{0}\right) e^{-a / \varepsilon}\right) A_{0} \\
+\beta_{1} e^{-\Psi^{\star} / \varepsilon} \sum_{j=1}^{N} u_{b}\left(s_{j}\right)\left|\nabla \Psi\left(\mathbf{x}_{j}\right)\right|\left(\tilde{\Psi}^{\prime \prime}\left(s_{j}\right)\right)^{-1 / 2}, \text { in Case 1, }
\end{gathered}
$$

$$
A_{0}^{\prime} \sim-\left(\lambda_{0}-\varepsilon^{\nu} g\left(\mathbf{x}_{0}\right) e^{-a / \varepsilon}\right) A_{0}+\beta_{2} e^{-\Psi^{\star} / \varepsilon} \sum_{j=1}^{N} u_{b}\left(s_{j}\right)\left|\nabla \Psi\left(\mathbf{x}_{j}\right)\right| d_{j}, \quad \text { in Case 2, }
$$

(4.20c) $A_{0}^{\prime} \sim-\left(\lambda_{0}-\varepsilon^{\nu} g\left(\mathbf{x}_{0}\right) e^{-a / \varepsilon}\right) A_{0}+\beta_{3} e^{-\Psi^{\star} / \varepsilon} \int_{S} u_{b}(s)|\nabla \Psi| d s$, in Case 3,

with initial value $A_{0}(0) \sim u_{0}\left(\mathbf{x}_{0}\right)$. The three cases are precisely those described in $\S 4.1$. Here, $\mathbf{x}_{0}$ is the global minimum of $\Psi$ in $D \cup \partial D, \tilde{\Psi}^{\prime \prime}\left(s_{j}\right)$ is defined in $(4.9 \mathrm{~b})$, and $\lambda_{0}$ is given in (4.9), (4.10) and (4.11) in Cases 1, 2, and 3, respectively. In addition, we have labeled

$$
\beta_{1} \equiv\left(\frac{H\left[\Psi\left(\mathbf{x}_{0}\right)\right]}{2 \pi \varepsilon}\right)^{1 / 2}, \quad \beta_{2} \equiv \frac{\Gamma\left(\frac{1}{2 p}\right)}{2 \pi p} \varepsilon^{\frac{1}{2 p}-1}\left(H\left[\Psi\left(\mathbf{x}_{0}\right)\right]\right)^{1 / 2}, \quad \beta_{3} \equiv \frac{\left(H\left[\Psi\left(\mathbf{x}_{0}\right)\right]\right)^{1 / 2}}{2 \pi \varepsilon},
$$

where $H$ is the Hessian. Moreover, $\mathbf{x}_{j} \in \partial D$ for $j=1,2, \ldots, N$ are those points where $\Psi$ is minimized on $\partial D$ with minimum value $\Psi^{\star} \equiv \Psi\left(\mathbf{x}_{j}\right)$ for $j=1,2, \ldots, N$, and $d_{j}$ defined by $\tilde{\Psi}(s)-\tilde{\Psi}\left(s_{j}\right)=d_{j}^{-2 p}(1+o(1))$ as $s \rightarrow s_{j}$ is a coefficient of the Taylor expansion of $\tilde{\Psi}(s)=\Psi\left(x_{1}(s), x_{2}(s)\right)$ at a degenerate minimum $\mathbf{x}_{j}$.

In summary, for $\varepsilon \rightarrow 0$ and $t \gg 1$, the metastable dynamics for (4.1) is given by $u(\mathbf{x}, t) \sim \tilde{u}^{\varepsilon}\left[\mathbf{x} ; A_{0}(t)\right]$ where $\tilde{u}^{\varepsilon}\left(\mathbf{x} ; A_{0}\right)$ is defined by (4.12) and $A_{0}(t)$ satisfies $(4.20)$. 
If $a$ is sufficiently large so that $\varepsilon^{\nu} g\left(x_{0}\right) e^{-\frac{a}{\varepsilon}}<\lambda_{0}$, then we obtain from (4.20) that $A_{0}(t) \rightarrow A_{0 e}$ as $t \rightarrow \infty$, where

$A_{0 e} \sim \sum_{j=1}^{N} u_{b}\left(s_{j}\right) \frac{\left|\nabla \Psi\left(\mathbf{x}_{j}\right)\right|}{\left[\tilde{\Psi}^{\prime \prime}\left(s_{j}\right)\right]^{1 / 2}}\left(\sum_{j=1}^{N} \frac{\left|\nabla \Psi\left(\mathbf{x}_{j}\right)\right|}{\left[\tilde{\Psi}^{\prime \prime}\left(s_{j}\right)\right]^{1 / 2}}-\frac{\varepsilon^{\nu} g\left(\mathbf{x}_{0}\right)}{\beta_{1}} e^{-\left(a-\Psi^{\star}\right) / \varepsilon}\right)^{-1}$ in Case 1,

$A_{0 e} \sim \sum_{j=1}^{N} u_{b}\left(s_{j}\right) d_{j} \mid \nabla \Psi\left(\mathbf{x}_{j} \mid\left(\sum_{j=1}^{N} d_{j}\left|\nabla \Psi\left(\mathbf{x}_{j}\right)\right|-\frac{\varepsilon^{\nu} g\left(\mathbf{x}_{0}\right)}{\beta_{2}} e^{-\left(a-\Psi^{\star}\right) / \varepsilon}\right)^{-1}\right.$ in Case 2,

(4.22c) $A_{0 e} \sim \int_{S} u_{b}(s)|\nabla \Psi| d s\left(\int_{S}|\nabla \Psi| d s-\frac{\varepsilon^{\nu} g\left(\mathbf{x}_{0}\right)}{\beta_{3}} e^{-\left(a-\Psi^{\star}\right) / \varepsilon}\right)^{-1}$ in Case 3 .

From (4.22) it is clear that $A_{0 e}$ is very sensitive with respect to changes in the value of $a$ when $a \approx \Psi^{\star}$.

When $g(\mathbf{x}) \equiv 0$ these results for $A_{0 e}$ were obtaned in [18] using the formal variational method of [11] (see also [11] for specific examples). The results for the timedependent and steady-state problems given in (4.20) and (4.22) when $g(\mathbf{x}) \neq 0$ are new. $\S 4.2$.

4.3. Two Examples. We now consider two examples of the theory of $\S 4.1$ and

EXAmple 4.1: Let $\mathbf{x}=(x, y)$ and let $D$ be the ellipse $x^{2} / 4+y^{2}=1$. Let $\Psi(\mathbf{x})=x^{2}+y^{2}$. Then, the minimum of $\Psi$ over $\bar{D}$ occurs at $\mathbf{x}_{0}=\mathbf{0}$. At this point, we calculate $H\left[\Psi\left(\mathbf{x}_{0}\right)\right]=4$ so that from $(4.21)$ we get $\beta_{1}=[2 /(\pi \varepsilon)]^{1 / 2}$.

For this example, there are two points on $\partial D$ that are closest to $\mathbf{x}_{0}=\mathbf{0}$. They are located at $\mathbf{y}_{1}=(0,1)$ and $\mathbf{y}_{2}=(0,-1)$. At these points, the curvatures $\kappa_{1}$ and $\kappa_{2}$ are $\kappa_{1}=\kappa_{2}=-1 / 4$. Then, from $(4.9 \mathrm{~b})$, we calculate $\tilde{\Psi}^{\prime \prime}\left(s_{j}\right)=3 / 2$ for $j=1,2$. Since $\Psi^{*}=1$, the estimate $(4.9 \mathrm{a})$ for $\lambda_{0}$ when $\varepsilon \ll 1$ reduces to

$$
\lambda_{0} \sim 8(3 \pi \varepsilon)^{-1 / 2} e^{-1 / \varepsilon} .
$$

For $t \gg 1$, we obtain from (4.12) and (4.20) that

$$
u(\mathbf{x}, t) \sim A_{0}(t)+\left[u_{b}(s)-A_{0}(t)\right] e^{\gamma \eta / \varepsilon}, \quad \gamma=\left.\gamma(s) \equiv \nabla \Psi \cdot \hat{n}\right|_{\partial D}>0,
$$

where $A_{0}(t)$ satisfies the limiting differential equation

$$
A_{0}^{\prime} \sim-8(3 \pi \varepsilon)^{-1 / 2} e^{-1 / \varepsilon}\left[1-\left(\frac{\sqrt{3 \pi}}{8}\right) \varepsilon^{\nu+1 / 2} g(\mathbf{0}) e^{-(a-1) / \varepsilon}\right]\left(A_{0}-A_{0 e}\right) .
$$

From (4.22a), the steady-state solution is

$$
A_{0 e} \sim \frac{1}{2}\left[u_{b}\left(\mathbf{y}_{1}\right)+u_{b}\left(\mathbf{y}_{2}\right)\right]\left[1-\left(\frac{\sqrt{3 \pi}}{8}\right) \varepsilon^{\nu+1 / 2} g(\mathbf{0}) e^{-(a-1) / \varepsilon}\right]^{-1} .
$$

Now, from (4.25), we observe that $A_{0}(t) \rightarrow A_{0 e}$ as $t \rightarrow \infty$ if and only if

$$
\left(\frac{\sqrt{3 \pi}}{8}\right) \varepsilon^{\nu+1 / 2} g(\mathbf{0}) e^{-(a-1) / \varepsilon}<1
$$


Thus, if $\nu=-1 / 2$ and $a>1$, then for $\varepsilon \rightarrow 0$ we have that $A_{0}(t) \rightarrow A_{0 e}$ as $t \rightarrow \infty$ for any initial condition $A_{0}(0)$. However, if $\nu=-1 / 2$ and $0<a<1$, then $A_{0}(t) \rightarrow A_{0 e}$ as $t \rightarrow \infty$ only when $g(\mathbf{0}) \leq 0$. In the borderline case where $a=1$ and $\nu=-1 / 2$, then

$$
\lim _{t \rightarrow \infty} A_{0}(t)=A_{0 e}, \quad \text { when } g(\mathbf{0})<8(3 \pi)^{-1 / 2}
$$

and $A_{0}(t) \rightarrow \infty$ as $t \rightarrow \infty$ when $g(\mathbf{0})>8(3 \pi)^{-1 / 2}$. Notice that when $a>1$, then

$$
A_{0 e} \sim \frac{1}{2}\left[u_{b}\left(\mathbf{y}_{1}\right)+u_{b}\left(\mathbf{y}_{2}\right)\right]
$$

is the average of the boundary data at the two closest points. This example clearly shows that $A_{0}(t)$ and $A_{0 e}$ depend supersensitively on $a$ when $a \approx 1$ and $\nu=-1 / 2$.

\begin{tabular}{|l|c|c|}
\hline \hline$\varepsilon$ & $A_{0 e}$ (asympotics) & $A_{0 e}$ (numerics) \\
\hline 0.15 & 0.47179 & 0.37496 \\
0.14 & 0.47179 & 0.37727 \\
0.13 & 0.47179 & 0.38061 \\
0.12 & 0.47179 & 0.38436 \\
0.11 & 0.47179 & 0.38879 \\
0.10 & 0.47179 & 0.39431 \\
0.09 & 0.47179 & 0.39943 \\
0.08 & 0.47179 & 0.40421 \\
0.07 & 0.47179 & 0.41240 \\
0.06 & 0.47179 & 0.41274 \\
0.05 & 0.47179 & 0.43011 \\
\hline
\end{tabular}

TABLE 4.1

Numerical. results (using PLTMG) of the constant $A_{0 e}$ for Example 4.2 for various $\varepsilon$ when $a=1 / 2$. The other parameter values are as given in Example 4.2.

EXAmPle 4.2 : Let $\mathbf{x}=(x, y)$ and $D$ be the square $-1<x<1,-1<y<1$. We let $\Psi=\left(x^{2}+y^{2}\right) / 2, g(\mathbf{x}) \equiv-1$, and $\nu=-1 / 2$. Then, there are four points on $\partial D$ that are closest to the minimum point $\mathbf{x}_{0}=0$ of $\Psi$. These points are labeled by $\mathbf{y}_{1}=(1,0), \mathbf{y}_{2}=(-1,0), \mathbf{y}_{3}=(0,1)$, and $\mathbf{y}_{4}=(0,-1)$. We calculate $|\nabla \Psi|$ and $\tilde{\Psi}^{\prime \prime}\left(s_{j}\right)=1$ at each of these boundary points. Also, $\Psi^{*}=1 / 2$ and $H[\Psi(\mathbf{0})]=1$, so that from (4.21), $\beta_{1}=(2 \pi \varepsilon)^{-1 / 2}$. Thus, from (4.9a), (4.20), and (4.22a), we obtain that

$$
\begin{gathered}
\lambda_{0} \sim 4(2 \pi \varepsilon)^{-1 / 2} e^{-\varepsilon^{-1} / 2}, \\
A_{0 e} \sim \frac{\sum_{j=1}^{4} u_{b}\left(\mathbf{y}_{j}\right)}{4}\left[1+\frac{\sqrt{2 \pi}}{4} e^{-(a-1 / 2) / \varepsilon}\right], \\
A_{0}^{\prime} \sim-4(2 \pi \varepsilon)^{-1 / 2} e^{-\varepsilon^{-1} / 2}\left[1+\frac{\sqrt{2 \pi}}{4} e^{-(a-1 / 2) / \varepsilon}\right]\left(A_{0}-A_{0 e}\right) .
\end{gathered}
$$

Finally, we took the boundary data $u_{b}=\sin ^{2}(2 x+y)$.

For this example, we used the finite element package PLTMG [5] to obtain full numerical results for the steady-state value $A_{0 e}$. When $a=1 / 2$, the numerical results 
for $A_{0 e}$ computed from PLTMG are compared in Table 4 for various values of $\varepsilon$ with the corresponding asymptotic result $(4.30 \mathrm{~b})$. We observe that the asymptotic and numerical results become closer only when $\varepsilon$ is rather small. We expect that when $\varepsilon<0.05$ the asymptotic and numerical results for $A_{0 e}$ would compare more favorably than for the larger values of $\varepsilon$ shown in Table 4 . However, we were unable to verify this prediction conclusively since, due to the exponentially small eigenvalue $\lambda_{0}$, PLTMG failed to converge when $\varepsilon<0.05$. Finally, in Table 5, where we chose $\varepsilon=0.07$, the asymptotic and numerical results for $A_{0 e}$ are compared for different values of $\nu$ and $a$. We would expect a more favorable agreement between the asymptotic and numerical results if we were able to compute solutions with PLTMG at a smaller value of $\varepsilon$. However, Table 5 does indeed show the supersensitivity of $A_{0 e}$ to $\nu$ and $a$ when $\varepsilon$ is small.

\begin{tabular}{|l|c|c|}
\hline \hline$(\nu, a)$ & $A_{0 e}$ (asympotics) & $A_{0 e}$ (numerics) \\
\hline$(-0.5, .75)$ & 0.754160 & 0.687259 \\
$(-0.5,1.0)$ & 0.767067 & 0.701080 \\
$(-0.5,1.25)$ & 0.767437 & 0.701101 \\
$(-0.5,2.0)$ & 0.767448 & 0.701112 \\
$(-0.5,4.0)$ & 0.767448 & 0.701138 \\
$(-.25,0.5)$ & 0.580374 & 0.512519 \\
$(0.0,0.5)$ & 0.658303 & 0.589825 \\
$(0.5,0.5)$ & 0.735197 & 0.667597 \\
$(1.0,0.5)$ & 0.758643 & 0.691938 \\
$(2.0,0.5)$ & 0.766825 & 0.701273 \\
$(4.0,0.5)$ & 0.767445 & 0.701261 \\
\hline
\end{tabular}

Numerical results (using PLTMG) of the constant $A_{0 e}$ for Example 4.2 for various a and $\nu$ when $\varepsilon=0.7$. The other parameter values are as given in Example 4.2.

Acknowledgements. M. J. W thanks the support of NSERC grant 81541 . We would also like to thank Prof. J. Wei of the Chinese University of Hong Kong for inviting us to contribute a paper for these proceedings.

\section{REFERENCES}

[1] R.C. Ackerberg, R.E. O'Malley, JR., Boundary layer problems exhibiting resonance, Stud. Appl. Math., 49 (1970), pp. 277-295.

[2] N. Alikakos, P.W. Bates, G. Fusco, Slow motion for the Cahn-Hilliard equation in one space dimension, J. Diff. Equat., 90 (1991), pp. 81-135.

[3] A.S. BAKHVALOV, On the optimization of methods for solving boundary value problems with boundary layers, (in Russian) Zh. Vychisl. Mat. i Mat. Fis., 9 (1969), pp. 841-859.

[4] F. Bai, C.M. Elliott, A. Gardiner, A. Spence, A.M. Stuart, The viscous Cahn-Hilliard equation. Part I: computations. Nonlinearity, 8 (1995), pp. 131-160.

[5] R.E. BANK, PLTMG: a software package for solving elliptic partial differential equations, Users' guide 7.0, Frontiers in Applied Mathematics, 15, SIAM Publications, (SIAM), Philadelphia, PA, (1994).

[6] P.W. Bates, J. Xun, Metastable patterns for the Cahn-Hilliard equation: part 1 and 2, J. Diff. Equat., 111 (1994), pp. 421-457; J. Diff. Equat., 117 (1995), pp. 165-216.

[7] J. Carr, R. Pego, Metastable patterns in solutions of $u_{t}=\varepsilon^{2} u_{x x}-f(u)$, Comm. Pure Appl. Math., 42 (1989), pp. 523-576.

[8] P.P.N. DEGROEN, The nature of resonance in a singular perturbation problem of turning point type, SIAM J. Math. Anal., 11 (1980), pp. 1-22. 
[9] C.M. Elliott, D.A. FrenCH, Numerical studies of the Cahn-Hilliard equation for phase separation, IMA J. Appl. Math., 38 (1987), pp. 97-128.

[10] G. FusCo, J.K. HALE, Slow motion manifolds, dormant instability and singular perturbations, J. Dyn. Diff. Equat., 1 (1989), pp. 75-94.

[11] J. GRASman, B. MATKowsky, A variational approach to singularly perturbed boundary value problems for ordinary and partial differential equations with turning points, SIAM J. Appl. Math., 32 (1977), pp. 588-597.

[12] V.A. Gushchin, V.V. ShChENNiKov, On a monotone difference scheme of the second order, (in Russian), Z. Vycisl. Mat. i Mat. Fiz., 14 (1974), pp. 789-792.

[13] A.M. IL'IN, A difference scheme for a differential equation with a small parameter affecting the highest derivative, Mat. Zametki, 6 (1969), pp. 237-248 (in Russian).

[14] D. IRon, M.J. WARD, A metastable spike solution for a non-local reaction-diffusion model, SIAM J. Appl. Math., 60 (2000), pp. 778-802.

[15] R.B. Kellogg, A. Tsan, Analysis of some difference approximations for a singularly perturbed problem without turning points, Math. Comp., 32 (1978), pp. 1025-1039.

[16] J.-Y. LEE, M.J. WARD, On the asymptotic and numerical analysis of exponentially illconditioned singularly perturbed boundary value problems, Stud. Appl. Math., 94 (1995), pp. 271-326.

[17] D. LuDwIG, Persistence of dynamical systems under random perturbations, SIAM Review, 17 (1975), pp. 605-640.

[18] B.J. MATKOWSKy, Z. Schuss, The exit problem for randomly perturbed dynamical systems, SIAM J. Appl. Math., 33 (1977), pp. 365-382.

[19] B.J. MATKOWSKY, Z. SChUSS, Eigenvalues of the Fokker-Planck operator and the approach to equilibrium for diffusions in potential fields, SIAM J. Appl. Math., 40 (1981), pp. 242-254.

[20] NAG Fortran library Mark 17, routine LSODI, Numerical Algorithms Group Ltd. Oxford, United Kingdom (1995).

[21] R.E. O'MAlley, M.J. Ward, Exponential Asymptotics, Boundary Layer Resonance, and Dynamic Metastability, in 'Mathematics is for Solving Problems', (P. Cook et al. editors), SIAM publication, (1997), pp. 189-203.

[22] L.G. REYNA, M.J. WARD, On the exponentially slow motion of a viscous shock, Comm. Pure Appl. Math., 48 (1995), pp. 79-120.

[23] L.G. REYNA, M.J. WARD, Metastable internal layer dynamics for the viscous Cahn-Hilliard equation, Methods and Appl. of Anal., 2, No. 3, (1995), pp. 285-306.

[24] H.-G. Roos, M. Stynes, L. ToBiska, Numerical Methods for Singularly Perturbed Differential Equations - Convection-Diffusion and Flow Problems, Springer series in Computational Mathematics, Springer-Verlag, Berlin, Heidelberg, New York, 1996.

[25] G.I. ShishKin, Methods of constructing grid approximations for singularly perturbed boundary value problems: condensing grid methods, Russ. J. Numer. Anal. Math. Modelling, 7 (1992), pp. 537-562.

[26] R. SRinivasan, A variational principle for the Ackerberg-O'Malley resonance problem, Stud. Appl. Math., 79, (1988), pp. 271-289.

[27] X. Sun, Numerical analysis of an exponentially ill-conditioned BVP with applications to metastable problems, accepted, IMA J. Numer. Analysis, (2000).

[28] X. SUn, M.J. WARD, Metastability for a generalized burgers equation with applications to propagating flame fronts, Europ. Journal of Appl. Math., 10, No. 1, (1999), pp. 27-53.

[29] X. SUn, M.J. WARD, Metastability and pinning for convection-diffusion-reaction equations in thin domains, Methods and Appl. of Anal., 6, No. 4, (2000), pp. 451-476.

[30] X. SUn, M.J. WARD, The dynamics and coarsening of interfaces for the viscous Cahn-Hilliard equation in one-spatial dimension, Stud. Appl. Math., 105 (2000), pp. 203-234.

[31] R. Vulanović, Some improvements of the nonequidistant Engquist-Osher scheme, Appl. Math. Comput., 40 (1990), no. 2, part II, pp. 147-164.

[32] M. Williams, Another look at Ackerberg-O'Malley resonance, SIAM J. Appl. Math., 41 (1981), pp. 288-293. 
